\title{
Exclusive semileptonic $B$ decays: lattice results and dispersive bounds*
}

\author{
Laurent Lellouch ${ }^{\dagger}$ \\ Theory Division, CERN, CH-1211 Geneva 23, Switzerland \\ E-mail: 'Iaurent. lellouch@cern.chi'
}

ABstract: The current status of lattice and dispersive bound calculations for exclusive semileptonic $B$ decays is reviewed. Emphasis is placed on decays relevant for the measurement of the sides of the unitarity triangle determined by $\left|V_{u b}\right|$ and $\left|V_{c b}\right|$.

\section{Introduction}

Exclusive semileptonic decays of hadrons containing a $b$ quark play an important role in testing the Standard Model. They enable the measurement of two of the three sides of the unitarity triangle, determined by the Cabibbo-KobayashiMaskawa (CKM) parameters $\left|V_{u b}\right|$ and $\left|V_{c b}\right|$. However, such measurements require a quantification of the non-perturbative, strong interaction dynamics which modify the elementary coupling of the $b$ and resulting quark to the $W$ boson. Lattice QCD and dispersive bounds provide tools to quantify and constrain these non-perturbative effects from first principles ${ }^{1}$. Model independence is crucial for the unitarity-triangle tests of the Standard Model to be meaningful. Without it, it will be impossible to disentangle potential new physics effects from artefacts of the models used.

The talk is divided into two main parts: lattice results and dispersive bounds. I will begin the first part with a brief introduction to the systematic uncertainties of lattice calculations and to the different ways in which heavy quarks can

*CERN-TH/99-352, LAPTH-Conf-765/99, CPT99/P.3911.

${ }^{\dagger}$ Address from October, 1999: LAPTH, Chemin de Bellevue, B.P. 110, F-74941 Annecy-le-Vieux Cedex, France. On leave from: Centre de Physique Théorique, Case 907, CNRS Luminy, F-13288 Marseille Cedex 9, France.

${ }^{1}$ Lattice results relevant for leptonic decays, neutral $B$ meson mixing and related topics, also useful for constraining the unitarity triangle, are covered in Shoji Hashimoto's talk ['] ['] be studied on a discrete spacetime. I will then turn to lattice results relevant for heavy-to-light quark decays, including $B \rightarrow \pi \ell \nu, B \rightarrow \rho \ell \nu$ and $B \rightarrow K^{*} \gamma$. This will be followed by a discussion of heavy-to-heavy quark decays, focussing mainly on $B \rightarrow D^{(*)} \ell \nu$ decays $^{2}$. The second part will begin with a brief description of the methodology of dispersive bounds and its applications to heavyto-heavy semileptonic decays. It will continue with applications of dispersive bound techniques to heavy-to-light decays.

\section{Lattice Results}

Although lattice QCD provides a means of determining non-perturbative, strong-interaction effects to arbitrary accuracy from first principles, in practice the results suffer from a variety of uncertainties due to limitations in computing resources. The main ones are:

- Statistical errors, associated with the fact that the QCD path integral is evaluated using Monte-Carlo simulations. They are estimated with standard statistical techniques.

- Discretisation errors, associated with the fact that spacetime is a discrete mesh of points. They are particularly important for heavy quarks and are dealt with in a varieties of ways, as discussed in Section 12.1 .

\footnotetext{
${ }^{2}$ Other recent lattice reviews of some of the subjects covered here can be found in $12,3 y$. The last Heavy Flavour review of lattice results for semileptonic decays is $[4 \mid$.
} 
- Finite volume errors, associated with the fact that in lattice calculations, spacetime is a finite box. They are not significant for the quantities discussed here.

- Uncertainties associated with extrapolations in light quark masses. On present day lattices, light quarks $(u$ and $d$ ) must have masses larger than about $m_{s} / 2$ so that the associated pions do not feel the edges of the box. One must therefore extrapolate results from these larger masses to the physical $u$ and $d$ quark masses.

- Matching errors, associated with the matching of lattice results onto the continuum. This matching is necessary because ultraviolet modes are treated differently on the lattice than they are in continuum regularisations. While it can be performed perturbatively, with resulting perturbative uncertainties, more and more it is carried out non-perturbatively, eliminating it as a source of systematic error. This is the case in most nonperturbatively-improved calculations discussed below.

- Quenching errors, associated with the fact that in most calculations of semileptonic $B$ decays, the feedback of quark loops on the gauge fields is neglected. It is not anticipated that these effects be more than $\mathcal{O}(10 \%)$ here. They have only very recently begun to be taken into account for these decays $[\underline{\tilde{p}}]$.

\subsection{Heavy-light hadrons on the lattice}

Because the $b$ quark with mass $m_{b} \sim 5 \mathrm{GeV}$ has a compton wavelength which is small compared to typical lattice spacings, $a \sim(2-3 \mathrm{GeV})^{-1}$, it cannot be simulated directly as a relativistic quark on present day lattices. This has led to a variety of approaches, which we review now for the case of hadrons composed of a heavy quark and light degrees of freedom.

\subsubsection{Relativistic quarks}

Relativistic fermions are obtained from a discretisation of the euclidean Dirac action. For heavy quarks, the discretisation most commonly used is that of Wilson. In modern calculations, it is often $O(a)$-improved by use of a ShekholeslamiWohlert (SW) action. This means that for heavy quarks $Q$ of mass $m_{Q}$, discretisation errors are formally reduced from $\mathcal{O}\left(a m_{Q}\right)$ to $\mathcal{O}\left(\alpha_{s} a m_{Q}\right)$ if the improvement is performed at tree level, and to $\mathcal{O}\left(\left(\alpha_{s} a m_{Q}\right)^{2}\right)$ if it is non-perturbative.

Despite these improvements, simulating the $b$ quark directly would lead to uncontrollable discretisation errors. Therefore, what is done is to perform the calculations for a number of quark masses around that of the charm, where discretisation errors are under control, and then extrapolate the results to $m_{b}$ using Heavy Quark Effective Theory (HQET) as a guide. Typically, HQET predicts that a form factor $F$ will scale with $m_{Q}$ as

$$
F(w) m_{Q}^{\nu}=A(w)\left(1+\frac{B(w)}{m_{Q}}+\mathcal{O}\left(\frac{1}{m_{Q}^{2}}\right)\right)
$$

at fixed four-velocity recoil $w \sim 1$, up to calculable logarithmic corrections. Here, $\nu, A$ and $B$ depend on the form factor.

The main problem with this approach is this rather long extrapolation to $m_{b}$.

\subsubsection{Effective theories}

The point of view here is that for heavy quarks whose mass $m_{Q}$ is large compared to typical QCD scales, $\mu_{Q C D}$, an expansion of QCD in powers of $\mu_{Q C D} / m_{Q}$ may be useful. Indeed, it reduces discretisation errors from powers of $a m_{Q}$ to powers of $a \mu_{Q C D}$, the latter being small on present day lattices.

Static quarks. The first implementation of this idea was to consider heavy quarks as static, spin- $1 / 2$ color sources. The problem with this approach is that accurate results for the physical $b$ quark require that $1 / m_{b}$-corrections be taken into account. This leads to power divergences proportional to inverse power of the lattice spacing which are difficult to subtract. It also leads to a proliferation of operators whose matrix elements must be computed. It has the further disadvanted of yielding correlators with poor signal to noise ratios.

"Non-relativistic QCD (NRQCD)", in the context of heavy-light mesons, essentially corresponds to keeping in the action some of the leading $1 / m_{Q^{-}}$ corrections mentioned above, thereby implicitly re-summing to infinite order their effects on the processes studied. The problem with this ap- 
proach is again power divergences and operator proliferation.

In the hybrid or "Fermilab" approach, the goal is to find an action valid for all $a m_{q}$. In practice, it is a relativistic SW action that is used and the results are subsequently given a nonrelativistic interpretation. There is debate over the extent to which systematics are controlled in this approach.

\subsection{Heavy-to-light decays}

In heavy-to-light-quark semileptonic decays, the light, final state hadron can have momenta as large as $|\vec{p}| \sim m_{Q} / 2$ in the parent rest frame. For $m_{Q}=m_{b}$, and on present day lattices, such momenta would lead to uncontrollably large discretisation effects proportional to powers of $a|\vec{p}|$. Therefore, at present, only a limited kinematical range near the zero-recoil point can be reached without extrapolation. Even so, lattice calculations are useful, for the relevant matrix elements are not normalised by heavy quark symmetry as they are for heavy-to-heavy quark decays (at zero recoil). Furthermore, experiment is beginning to measure the corresponding differential rates within the lattice's kinematical reach [6] will allow model-independent determinations of CKM parameters such as $\left|V_{u b}\right|$. Finally, lattice groups are investigating new ways of exploring the region of large recoils.

\subsection{1 $B^{0} \rightarrow \pi^{-} \ell^{+} \nu$}

In the past two years, most studies of heavy-tolight decays have concentrated on $B^{0} \rightarrow \pi^{-} \ell^{+} \nu$. The relevant matrix element is

$$
\begin{aligned}
& \left\langle\pi^{-}\left(p^{\prime}\right)\left|V^{\mu}\right| B^{0}(p)\right\rangle=\frac{M_{B}^{2}-M_{\pi}^{2}}{q^{2}} q^{\mu} f_{0}\left(q^{2}\right) \\
& +\left(p+p^{\prime}-\frac{M_{B}^{2}-M_{\pi}^{2}}{q^{2}} q\right)^{\mu} f_{+}\left(q^{2}\right),
\end{aligned}
$$

where $q=p-p^{\prime}$ and $V^{\mu}=\bar{b} \gamma^{\mu} u$.

In Figure 1], I have collected results for both

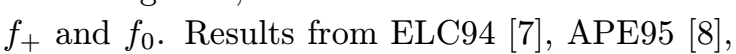

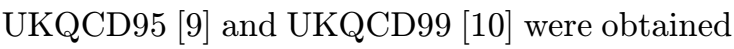
using relativistic quarks. APE95 and UKQCD95 implement tree-level improvement, while UKQCD99 implements full non-perturbative improvement. The FNAL99 [1] $\left.\overline{1}_{1}^{1}, \overline{1} \overline{2}_{1}^{\prime}\right]$ results were obtained with the hybrid approach while HiKEK98
[13.] use NRQCD. Besides the non-perturbative improvement of UKQCD99 and the fact that FNAL99 has begun exploring cutoff dependence by performing their calculation at three values of the lattice spacing ${ }^{3}$, another novelty is the fact that FNAL99 and UKQCD99 were able to extrapolate the form factors in light quark mass to the physical $u$ and $d$ masses. APE95 and ELC94 had only performed this extrapolation for a single $q^{2}$. As indicated by the "no $\chi$ ", HiKEK98 and UKQCD95 have $u$ and $d$ quarks with masses around that of the strange. A systematic uncertainty has been added to these results to account for this fact, as described in [1] in. It should be noted that UKQCD99's error bars include a wide range of systematics. Their results are collected in Table

Despite the wide range of approaches, results for both form factors are generally consistent. This is rather reassuring given the number of fits and extrapolations involved. Also plotted in Figure '1', are the light-cone sumrule (LCSR) results

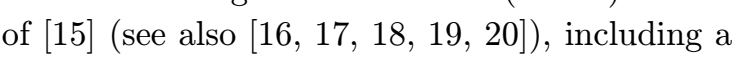
$20 \%$ error band. In the region of overlap, agreement is excellent. Furthermore, the LCSR curves do look like natural extensions of the lattice results.

The differential decay rate is easily obtained from the form factors, up to an overall factor of $\left|V_{u b}\right|^{2}$. Therefore, comparison with experiment in the range of pion momenta reached by the lattice calculation will yield a model-independent determination of $\left|V_{u b}\right|$. FNAL suggest to compare $d \Gamma / d\left|\vec{p}_{\pi}\right|$ in the range $0.4 \mathrm{GeV} \leq\left|\overrightarrow{p_{\pi}}\right| \leq$ $0.8 \mathrm{GeV}$, where their systematic errors are minimised $\left[1 \overline{1} 1,1, \overline{1}{ }_{1}^{1}\right]$. Their rate is shown in Figure 2' together with that of UKQCD99. Agreement is good, a bit less so at the lowest value of $\left|\overrightarrow{p_{\pi}}\right|$, where sensitivity to the $B^{*}$ pole is strongest. The results of UKQCD99 for $d \Gamma / d q^{2}$ for $0.5 \mathrm{GeV} \leq$ $\left|\overrightarrow{p_{\pi}}\right| \leq 1.1 \mathrm{GeV}$, with an estimate of systematic errors, are given in Table ${ }_{1}^{1}$.

One may also attempt to extrapolate the above lattice results to the large recoil region. While it can be done in a model-independent way as described in Section 3.2 , extrapolation of the most

${ }^{3}$ The FNAL99 results shown here are those obtained at $\beta=5.9$. 

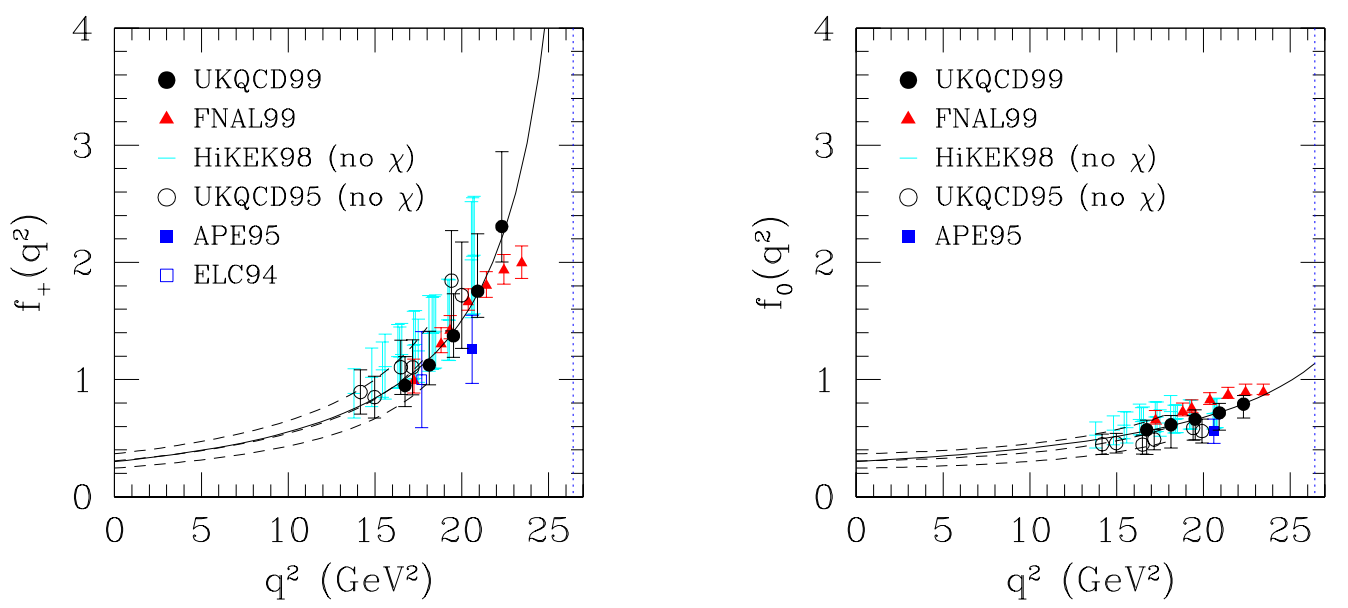

Figure 1: Form factors for $B^{0} \rightarrow \pi^{-} \ell^{+} \nu$. The points are results from various lattice groups. The dashed lines are LCSR results (see text). The solid lines are the result of the fit of UKQCD99's central value results, with statistical errors only, to the BK parameterisation of Eq. (i2.3i) [1]

\begin{tabular}{|c|c|c|c|c|c|}
\hline$q^{2}(\mathrm{GeV})^{2}$ & 16.7 & 18.1 & 19.5 & 20.9 & 22.3 \\
\hline$f_{+}\left(q^{2}\right)$ & $0.9_{-2}^{+1}+2$ & $1.1_{-2}^{+2}{ }_{-1}^{+2}$ & $1.4_{-2}^{+2}{ }_{-1}^{+3}$ & $1.8_{-2}^{+2}+4$ & $2.3_{-3}^{+3}{ }_{-1}^{+6}$ \\
\hline$f_{0}\left(q^{2}\right)$ & $0.57_{-6}^{+6}+5$ & $0.61_{-6}^{+6}+6$ & $0.66_{-5}^{+5}+6$ & $0.72_{-4-14}^{+5}+6$ & $0.79_{-4-11}^{+5}+6$ \\
\hline $1 /\left|V_{\mathrm{ub}}\right|^{2} \mathrm{~d} \Gamma / \mathrm{d} q^{2}\left(\mathrm{ps}^{-1} \mathrm{GeV}^{-2}\right)$ & $0.29_{-9}^{+10+11}$ & $0.27_{-7}^{+8}{ }_{-1}^{+1}$ & $0.25_{-6}^{+7}+11$ & $0.23_{-5}^{+6}+11$ & $0.20_{-5}^{+5}+9$ \\
\hline
\end{tabular}

Table 1: Form factors and differential decay rate as functions of $q^{2}$ from UKQCD99 [i10].

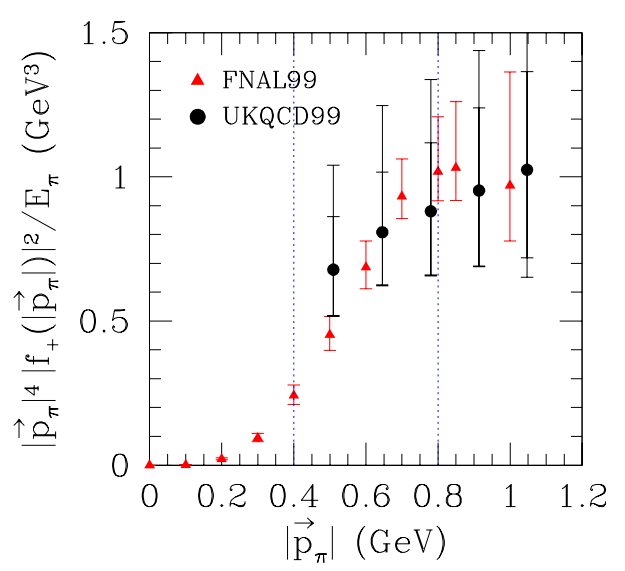

Figure 2: Differential decay rate for $B^{0} \rightarrow \pi^{-} \ell^{+} \nu$

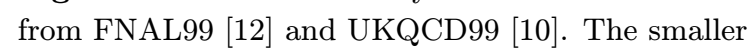
error bars on the UKQCD99 results are statistical; the larger ones are statistical and systematic combined in quadrature.

recent results has only been performed using models. It is not, therefore, on the same firm theoretical ground as the unextrapolated results. Nevertheless, it may be useful if one needs information about the decay over the full kinematical range ${ }^{4}$. UKQCD99 has considered fits to

$$
\begin{aligned}
f_{+}\left(q^{2}\right) & =\frac{f(0)}{\left(1-q^{2} / M_{B^{\star}}^{2}\right)\left(1-\alpha q^{2} / M_{B^{\star}}^{2}\right)} \\
f_{0}\left(q^{2}\right) & =\frac{f(0)}{\left(1-q^{2} / \beta M_{B^{\star}}^{2}\right)},
\end{aligned}
$$

with either $\alpha=1$ and $f(0), \beta$ as fit parameters (dipole/pole), or leaving all three parameters free, as suggested by Becirevic and Kaidalov (BK) [ $\left[22^{\prime}\right]$. These two parameterisations are consistent with the kinematical constraint $f_{+}(0)=$ $f_{0}(0)$, heavy-quark scaling at large $q^{2}$ (Eq. (2.1) and light-cone (LC) scaling at small $q^{2}$ (Eq. (2.6-1)

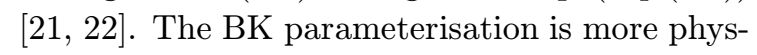
ical in that it correctly takes into account the $B^{*}$ pole contribution to $f_{+}$. It is important to use parameterisations which are consistent with as many model-independent constraints as possible.

The result of UKQCD99's fit to the BK parameterisation is shown in Figure $\overline{1}$ 1.'. Agreement $\left.\begin{array}{lll}{ }^{4} \text { Please see }\left[g^{\prime},\right. & 21\end{array}\right]$ for earlier constrained $q^{2}$ 


\begin{tabular}{|c|c|c|c|}
\hline & Rate & $f_{+, 0}(0)$ & description \\
\hline UKQCD99 [10 & $9_{-2-4}^{+3+3}$ & $0.30_{-4-9}^{+6+4}$ & BK fit plus many systematics \\
\hline APE99 [29! & & $0.28(4)$ & LC scaling at $q^{2}=0$ \\
\hline UKQCD98 ["in & $8.5_{-1.4}^{+3.3}$ & $0.27(11)$ & dipole/pole fit to $f_{+} / f_{0}$ \\
\hline APE95 ["8;] & $8 \pm 4$ & $0.35(8)$ & $q^{2} \simeq 20.4 \mathrm{GeV}^{2}$, pole fit, $m_{\mathrm{p}}=5.32(1) \mathrm{GeV}$ \\
\hline ELC94 [7] & $9 \pm 6$ & $0.30(14)(5)$ & $q^{2} \simeq 18 \mathrm{GeV}^{2}$, pole fit, $m_{\mathrm{p}}=5.29(1) \mathrm{GeV}$ \\
\hline
\end{tabular}

Table 2: $f_{+, 0}$ at $q^{2}=0$ and total rate in units of $\left|V_{u b}\right|^{2} \mathrm{ps}^{-1}$. The results of APE and ELC are obtained by extrapolation of $f_{+}$determined at a single $q^{2}$ value, as indicated in column 4. UKQCD uses results for $f_{+}$ and $f_{0}$ at a number of $q^{2}$ values and performs constrained fits to the parameterisations of Eq. (2.3) (2.3). The final state pion in the UKQCD98 results is composed of quarks with masses around that of the strange and include a systematic error to account for this. The APE99 results was obtained as described around Eq. (12.6.6.

with the LCSR results is stunning. UKQCD99 then uses its fits to obtain the total rate. Their result, along with older lattice results, is given in Table ${ }_{2}^{2}-i$ Agreement is good, though error bars are large.

While the extrapolations discussed above introduce model-dependence, there are many possible checks and constraints. For instance, the residue of the $B^{*}$ pole in $f_{+}$is related to the coupling of the pion to heavy mesons, defined in Eq. $(2.7):$

$$
f_{\mathrm{res}} \equiv \frac{\operatorname{Res}_{q^{2}=m_{B^{*}}^{2}} f_{+}\left(q^{2}\right)}{M_{B^{*}}^{2}}=\frac{g_{B^{*} B \pi}}{2 f_{B^{*}}}
$$

where $f_{B^{*}}$ is given by $\left\langle 0\left|V_{\mu}\right| B^{*}\right\rangle=\varepsilon_{\mu} M_{B^{*}}^{2} / f_{B^{*}}$. Thus, to the extent that the BK parameterisation is a valid description of $f_{+}$and $f_{0}$, the direct determination of this residue, $f_{\text {res }}=f(0) /(1-$ $\alpha)$, must agree with its indirect determination through $g_{B^{*} B \pi}$ and $f_{B^{*}}$. The results of UKQCD99 yield $f_{\text {res }}=0.55_{-7-0}^{+7+51}$ while Eq. (2.4) with $f_{B^{*}}=27_{-3-5}^{+3+0}$ from $[23]$ and $g$ from Eq. (2.9i) give $f_{\text {res }}=0.63_{-10-12}^{+10+17}$. Error bars are large and $g$ from Eq. (2.9) is exploratory and was converted to $g_{B^{*} B \pi}$ using Eq. (2.81) without $1 / m_{b^{-}}$ corrections. Agreement within statistical errors, though, indicates a certain consistency of the BK fit to the central values. Furthermore, statistical and systematic uncertainties on the indirect determination are comparable to or smaller than those on the direct determination. This suggests that some gain may result from constraining the $B^{*}$-pole residue with its indirect value. Of course, for this to be done correctly would require a calculation of the form factors, as well as
$f_{B^{*}}$ and $g_{B^{*} B \pi}$, with the same lattice parameters.

Another constraint comes from a soft pion theorem for $f_{0}$ which states that

$$
f_{0}\left(q_{\max }^{2}\right)=\frac{f_{B}}{f \pi}
$$

in the chiral limit [24, 25$]$. There is some controversy as to whether the theorem is upheld by the lattice results (see, for instance, issue should be resolved within the coming year.

Further constraints, as we have already mentioned, come from the scaling of heavy-to-light form factors with the mass of the heavy quark and the energy of the final state meson (in the heavy-meson frame), in the combined heavy-quark

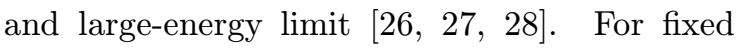
$q^{2} \sim 0$, this scaling becomes a universal scaling of all form factors with the heavy-meson mass:

$F\left(q^{2}\right) M_{B}^{3 / 2}=A\left(q^{2}\right)\left(1+\frac{B\left(q^{2}\right)}{M_{B}}+\mathcal{O}\left(\frac{1}{M_{B}^{2}}\right)\right)$,

where $F\left(q^{2}\right)$ is a generic heavy-to-light form factor. APE99 [2" $\overline{2}$ ] has used the parameterisation of Eq. (2.6i) to extrapolate $f_{+, 0}(0)$ in heavy-quark mass from around the charm, where the lattice yields the form factors for a range of $q^{2}$ that encompasses zero, up to the $b$. This extrapolation is shown in Figure and the resulting value given in Table $1 / M_{B}$-corrections in Eq. (2.6i) can be taken into account, as shown in the figure. The value reported is consistent with the one obtained from the results of UKQCD99 as well as with older results, as shown in Table couraging, for it suggests that $B$-to-light form 
factors could be reconstructed in the large recoil region from the lattice $D$-to-light form factors, in a model-independent way. A couple words of warning, however: LCSR estimates $\left[2 \overline{8}^{\prime \prime}{ }^{2} \overline{2} \bar{O}_{n}^{\prime \prime}\right.$, as well as the figure, suggest that pre-asymptotic corrections in Eq. $(2 . \overline{6})$ are large, even at $M_{B}$, and one may worry about the convergence of the expansion in the region where lattice results are available. Theoretical work is also needed to understand the expansion of Eq. (20.6) better.

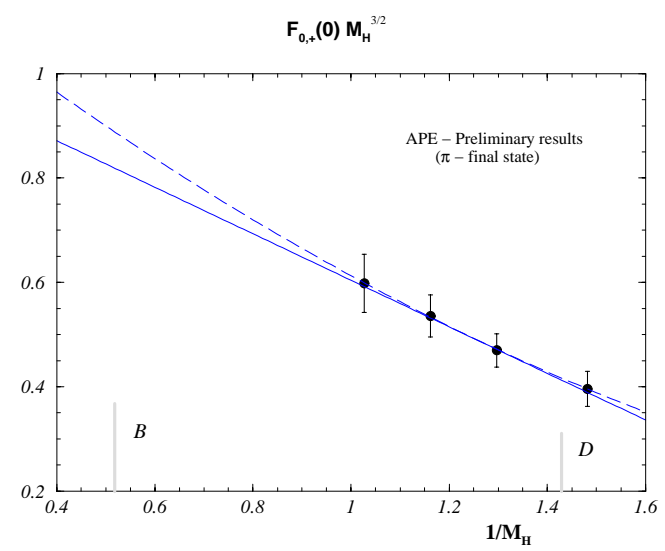

Figure 3: APE99's LC scaling of $f_{+, 0}(0)$ from heavy hadron mass $M_{H} \sim M_{D}$ to $M_{B}$, according to Eq. $\left.(2.6)^{n}\right)$ [29in. The points are the simulation results. The curves are the result of a linear (solid) and a quadratic (dashed) fit to Eq. (․․․․

Before closing this section, it should be mentioned that preliminary results of a calculation for $B \rightarrow \pi$ form factors with two flavours of dynamical quarks (partial unquenching) were pre-

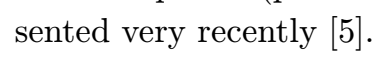

\subsubsection{First lattice determination of the $B^{*} B \pi$ coupling}

Another novelty since the last Heavy Flavour conference is a first lattice determination of the $B^{*} B \pi$ coupling, defined as the $q^{2} \rightarrow m_{\pi}^{2}$ limit of $g_{B^{*} B \pi}\left(q^{2}\right)$ in

$$
\begin{gathered}
\left\langle B^{0}(p) \pi^{+}(q) \mid B^{*+}\left(p^{\prime}\right)\right\rangle= \\
-g_{B^{*} B \pi}\left(q^{2}\right) q \cdot \eta(2 \pi)^{4} \delta\left(p^{\prime}-p-q\right) .
\end{gathered}
$$

As we have already seen in Eq. (2.4) , it is related to the residue of $f_{+}$at the $B^{*}$ pole. Furthermore, it determines the coupling, $g$, of $B, B^{*}$ and $\pi$ in
Heavy Meson Chiral lagrangians through

$$
g_{B^{*} B \pi}\left(q^{2} \rightarrow 0\right) \times \frac{f_{\pi}}{2 M_{B}} \stackrel{m_{b} \rightarrow \infty}{\longrightarrow} g .
$$

LSZ reduction and $\mathrm{PCAC}$ relate the matrix element of Eq. (12.7) to $\left\langle B^{0}(p)\left|A_{\mu}\right| B^{*+}(p+q)\right\rangle$. The authors of [31 1 compute the latter, and do so in the static quark limit. This exploratory study, performed with a rather large lattice spacing, yields

$$
g=0.42(4)(8)
$$

which compares favourably with the combined estimate of [32], $g \simeq 0.38(8)$ and is roughly consistent with the value, $g=0.27_{-2-2}^{+4+5}$, obtained from an analysis of the experimental measurement of $D_{(s)}^{*(0,+)} \rightarrow D_{(s)}^{(0,+)} \pi^{0}$ and the corresponding radiative decays $\left[33^{n}\right]^{5}$. It can also be compared to the value of $g$ obtained from the direct determination of the residue of $f_{+}$given below Eq. (2.4). Using Eq. (2.8 ing the value of $f_{B^{*}}$ given after Eq. (2.4) , I find $g=0.37_{-7-7}^{+7+34}$, consistent within statistical errors.

JLQCD are undertaking an indirect determination of $g$ as part of their NRQCD study of $B \rightarrow \pi \ell \nu$ decays, from the residue of the $B^{*}$ pole [2in] There are also determinations of $g$ from the residue of the $B^{*}$ pole from older lattice results in [1] 14 .

\subsection{3 $B \rightarrow \rho \ell \nu$ and $B \rightarrow K^{*} \gamma$}

Although no new lattice results for these decays have been obtained since the last Heavy Flavour conference, it is worth reviewing a few results. The definitions of the form factors discussed below can be found, for instance, in [34

UKQCD [34.] proposed obtaining $\left|V_{u b}\right|$ from a comparison of lattice and experimental results for the differential rate for $B^{0} \rightarrow \rho^{-} \ell^{+} \nu$ decays for $q^{2} \gtrsim 12 \mathrm{GeV}^{2}$. To that effect, they parameterised this rate as

$$
\frac{d \Gamma}{d q^{2}}=\frac{G_{F}^{2}\left|V_{u b}\right|^{2}}{192 \pi^{3} M_{B}^{3}} q^{2} \sqrt{\lambda\left(q^{2}\right)} a^{2}\left(1+b\left(q^{2}-q_{\max }^{2}\right)\right)
$$

\footnotetext{
${ }^{5}$ The author excludes a second possible solution, $g=$ $0.76_{-3-1}^{+3+2}$, at the two-sigma level with the experimental limit, $\Gamma_{D^{*}+}<13 \mathrm{MeV}$.
} 
where $\lambda\left(q^{2}\right)=\left(M_{B}^{2}+M_{\rho}^{2}-q^{2}\right)^{2}-4 M_{B}^{2} M_{\rho}^{2}$ and where the parameters $a$ and $b$ are determined by fitting to lattice results obtained for $q^{2} \gtrsim 14 \mathrm{GeV}^{2}$. They find

$$
\begin{aligned}
a & =4.6_{-0.3}^{+0.4} \pm 0.6 \mathrm{GeV} \\
b & =\left(-8_{-6}^{+4}\right) \times 10^{-2} \mathrm{GeV}^{-2}
\end{aligned}
$$

where $a$ includes a $6.5 \%$ systematic uncertainty associated with light spectator mass dependence and a $10 \%$ uncertainty associated with discretisation effects. $a$, here, plays the role of $\mathcal{F}_{D^{*}}(1)$ in the determination of $\left|V_{c b}\right|$ from $B \rightarrow D^{*} \ell \nu$ decays, and $b$ the role of $\mathcal{F}_{D^{*}}$ 's slope at $w=1$.

For $\rho=K^{*}$, heavy-quark spin symmetry predicts that the form factors $V$ and $A_{1}$ for $B \rightarrow$ $\rho \ell \nu$ and $T_{1,2}$ for $B \rightarrow K^{*} \gamma$ are related as [35-1]

$$
\begin{gathered}
\frac{V}{2 T_{1}}(w)=1+\mathcal{O}\left(\frac{1}{m_{b}}\right) \\
\frac{A_{1}}{2 i T_{2}}(w)=1+\mathcal{O}\left(\frac{1}{m_{b}}\right),
\end{gathered}
$$

for $w=v_{B} \cdot v_{\rho, K^{*}} \sim 1$. Results for these ratios are shown in Figure 4 , for different values of the initial heavy-meson mass and for a $\rho$ and $K^{*}$ composed of quarks slightly more massive than the strange. Both relations are well satisfied in the infinite heavy-quark-mass limit. The surprising result is that the ratio $A_{1} / 2 i T_{2}$ still satisfies the heavy-quark prediction, even at the $D$ mass. One can further include all $1 / m_{b}$ corrections from kinematics $\left[\overline{3} \overline{5}^{1}, \overline{3} \overline{6}_{0}^{1}, \overline{3} \overline{2} \overline{1}\right]$ in the ratios of Eq. (2.12i). With these corrections the relation between $V$ and $T_{1}$ is improved by a factor of about two while the relation between $A_{1}$ and $T_{2}$ is not significantly changed. Similar relations, including the $1 / m_{b}$ corrections from kinematics, have been considered with LCSR, but for large recoils $(1.7 \lesssim w \lesssim 3.5)$, and are found to hold to

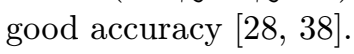

As already discussed for $B \rightarrow \pi \ell \nu$ decays, it may be of interest to abandon model-independence in favour of a simple description of the relevant form factors over the full kinematical range. UKQCD [2근, thus considered combined fits of lattice results for the form factors relevant for $B \rightarrow$ $\rho \ell \nu$ and $B \rightarrow K^{*} \gamma$ decays, obtained at high $q^{2}$. The parameterisations used are consistent with heavy-quark scaling at small recoils (Eq.
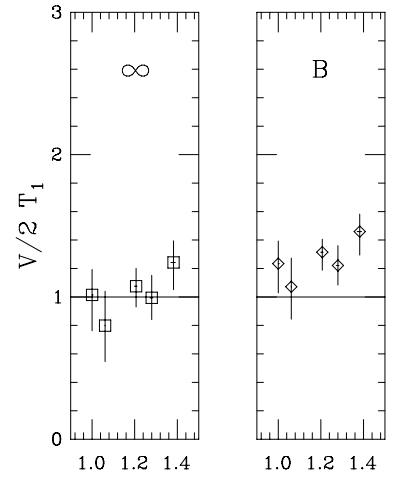

$\begin{array}{lll}1.0 & 1.2 & 1.4\end{array}$

$\omega$
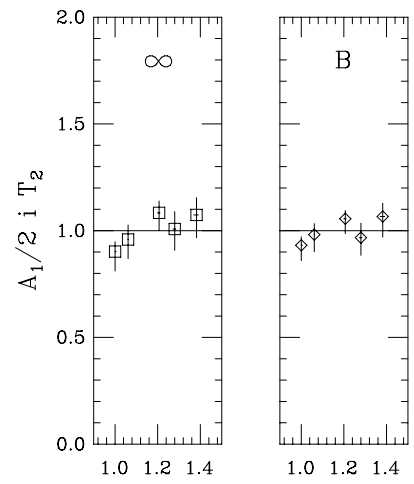

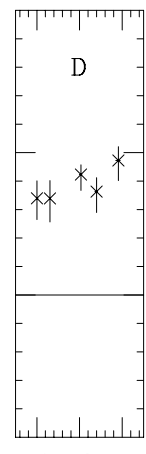

$\begin{array}{lll}1.0 & 1.2 & 1.4\end{array}$

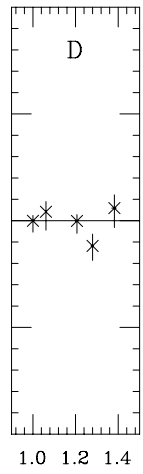

Figure 4: Ratios $V / 2 T_{1}$ and $A_{1} / 2 i T_{2}$ for five values of $w$ and three values of the initial heavy-meson mass [3] in]. The horizontal lines are the heavy-quark limit values.

(2.11)) and light-cone scaling at large recoils (Eq. $(2.6 i))$, as well as with the kinematical constraint at $q^{2}=0, T_{1}(0)=i T_{2}(0)^{6}$. For degenerate $\rho$ and $K^{*}$, leading order HQET predicts that the seven form factors required to parameterise the relevant matrix elements are related to four IsgurWise functions $\left[\overline{3} \overline{5}_{1}, \overline{3} \overline{7}_{1}\right]$. Keeping only one of these functions, which is fixed by parameterising $A_{1}\left(q^{2}\right)$ by a pole form with free pole position and residue, the authors of [21] obtain a twoparameter description of the seven form factors. The results of the fit to this parameterisation are shown in Figure 15 , for a final state $\rho^{7}$. Also shown

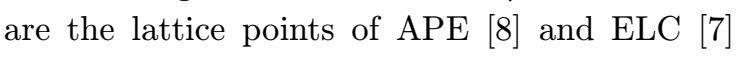
as well as the LCSR results of [38']. Agreement

\footnotetext{
${ }^{6}$ They also consider $B \rightarrow \pi \ell \nu$ decays, but the fits discussed around Eq. (2.3) are performed with improved lattice results.

${ }^{7} A_{2}\left(q^{2}\right)$ and $T_{3}\left(q^{2}\right)$ are not included in the fit. $T_{3}\left(q^{2}\right)$ has not yet been calculated.
} 

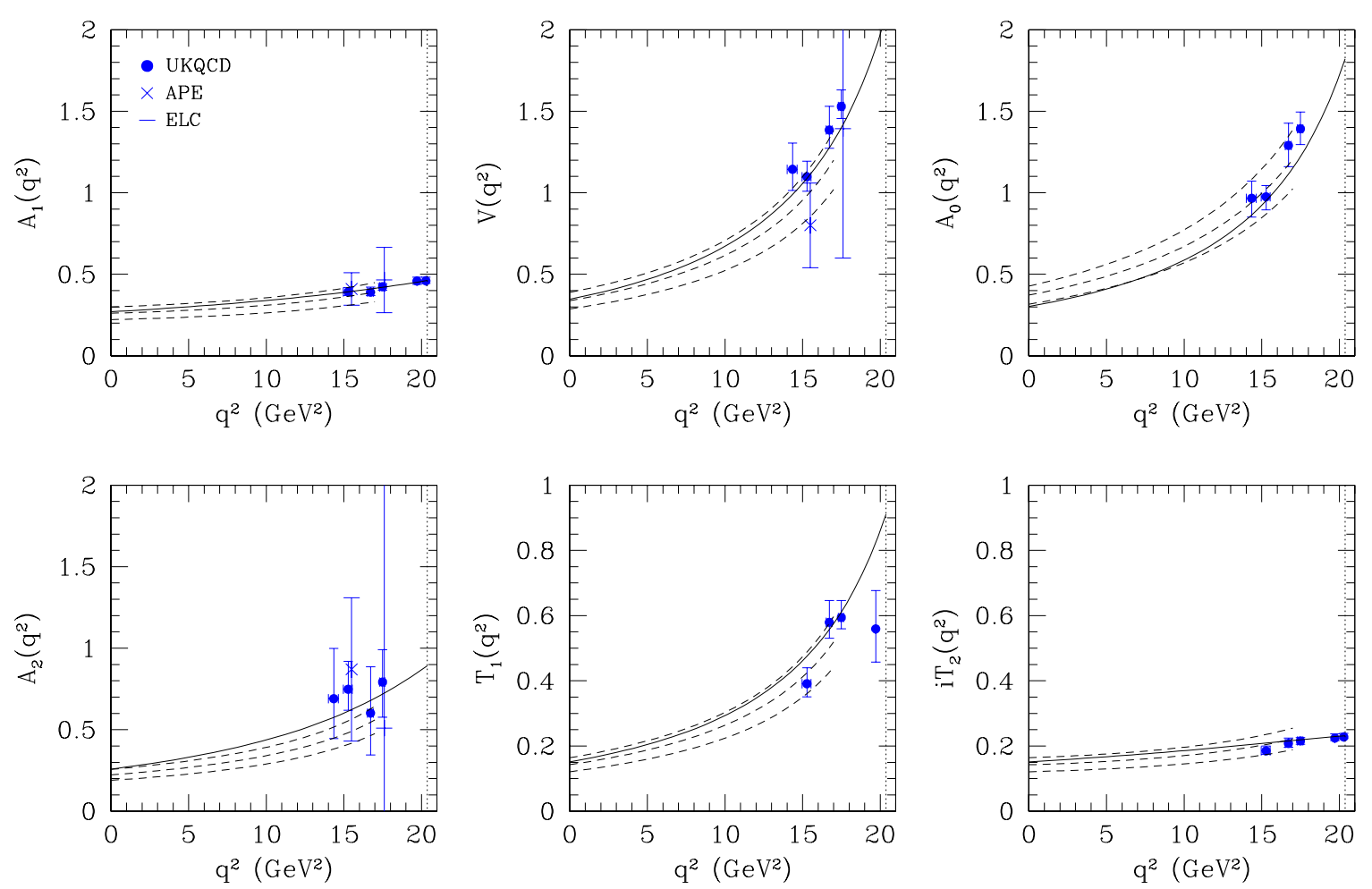

Figure 5: $B \rightarrow \rho$ form factors. The solid curves are fits of the UKQCD results to the constrained pa-

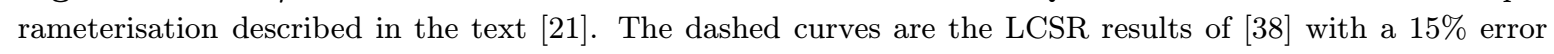
band.

with the lattice results in the region of overlap is encouraging as is the agreement at smaller $q^{2}$, with the lattice-constrained parameterisation. It is worth pointing out that the error bars on the UKQCD results are statistical only. As discussed in [34] cretisation errors and a 5-20\% uncertainty, depending on the form factor, associated with light spectator mass dependence. We also emphasise that the extrapolated form factors are no longer model independent, though models related to the one used have recently received theoretical support from the formalism developed in [2 $2 \overline{6}$ - $]$.

Similar results are obtained for a final state $K^{*}$ and agreement with LC sumrules is also good. These results enable a determination of $T_{1}(0)$ which determines the rate for $B \rightarrow K^{*} \gamma$. In Table $\overline{\beta_{1}}$ we compare $T_{1}(0)$ from the combined fit of $[21$, to older results obtained with less constrained extrapolations. The UKQCD value yields $R_{K^{*}}=\Gamma\left(B \rightarrow K^{*} \gamma\right) / \Gamma(b \rightarrow s \gamma)=16_{-4}^{+3} \%$, to leading order in $\alpha_{s}$ and up to $\mathcal{O}\left(1 / m_{B}^{2}\right)$ correc- tions $[\overline{3} \overline{3} \bar{j}$. Errors are statistical only. This result is consistent with the experimental result from CLEO [400"], $R_{K^{*}}=13(4) \%$.

\subsubsection{Other decays}

APE [209] is also considering the penguin induced matrix elements for $B \rightarrow K(\pi) \ell^{+} \ell^{-}$decays.

\subsection{Heavy-to-heavy decays}

The recoils involved in semileptonic heavy-to-heavy quark decays are much smaller than those in heavy-to-light decays: the lattice can cover the full kinematical range. Heavy quark symmetry is also much stronger, here, in that it normalises the relevant matrix elements at zero recoil and applies to the full kinematical range. Nevertheless, the lattice can provide interesting information on the behaviour of these matrix elements away from zero recoil as well as provide tests of heavy quark symmetry. Attempts are also being made to quantify deviations from heavy-quark 


\begin{tabular}{rcl}
\hline & $T_{1}(0)$ & $T_{2}\left(q_{\max }^{2}\right)$ \\
\hline UKQCD98 [21, [1] & $0.16_{-1}^{+2}$ & $0.25(2)$ \\
LANL96 [41, & $0.09(1)$ & \\
APE96 [42] & $0.09(1)(1)$ & \\
BHS94 [43] & $0.101(10)(28)$ & $0.325(33)(65)$ \\
\hline
\end{tabular}

Table 3: $T_{2}\left(q_{\max }^{2}\right)$ is obtained using heavy-quark scaling from around the charm region to the $b$. For $T_{1}(0)=$

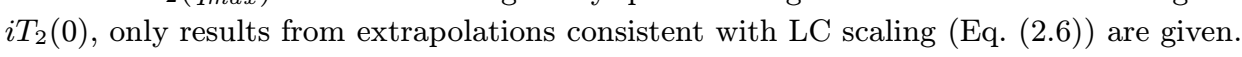

normalisations for physical quark masses at the zero-recoil point.

\subsection{1 $B \rightarrow D^{(*)} \ell \nu$ : form factors at zero re- coil}

To extract $\left|V_{c b}\right|$ from $B \rightarrow D^{(*)} \ell \nu$ decays, one traditionally extrapolates the differential decay rates to the zero-recoil point, $w=v_{B} \cdot v_{D^{(*)}}=1$, obtaining $\left|V_{c b}\right| \mathcal{F}_{D^{(*)}}(w=1)$. One then uses the fact that the form factors $\mathcal{F}_{D^{(*)}}(1)$ are equal to 1 in the heavy quark limit to obtain $\left|V_{c b}\right|$. A precise determination of $\left|V_{c b}\right|$, however, requires calculation of the corrections to this limit. Apart from calculable, perturbative corrections, there are non-perturbative corrections proportional to inverse powers of the heavy quark masses, $m_{c, b}$. Uncertainties on these power corrections currently limit a more precise determination of $\left|V_{c b}\right|$.

It was proposed recently that these corrections be obtained by studying the heavy-quarkmass dependence of the relevant form factors on the lattice [44], instead of evaluating, for instance, the contributions of subleading HQET operators in the $1 / m_{c, b}$ expansion. In [44], this idea is applied to the determination of power corrections to $\mathcal{F}_{D}(1)$. In $\left[\overline{4}_{2}^{4}\right]$, the same authors present preliminary results for the corrections to $\mathcal{F}_{D^{*}}(1)$. Since their method requires measuring small deviations from 1 , excellent control of both statistical and systematic errors is necessary. Thus, they suggest studying the mass dependence of double ratios of three-point functions which at asymptotic times reduce to a double ratio of zero-recoil matrix elements ${ }^{8}$ :

$R_{J_{\mu}}^{B^{(*)} \rightarrow D^{(*)}} \longrightarrow \frac{\left\langle D^{(*)}\left|J_{\mu}^{c b}\right| B^{(*)}\right\rangle\left\langle B^{(*)}\left|J_{\mu}^{b c}\right| D^{(*)}\right\rangle}{\left\langle B^{(*)}\left|J_{\mu}^{b b}\right| B^{(*)}\right\rangle\left\langle D^{(*)}\left|J_{\mu}^{c c}\right| D^{(*)}\right\rangle}$,

\footnotetext{
${ }^{8}$ To determine zero-recoil, power corrections in the form factor $h_{-}(w)$, relevant for $B \rightarrow D \ell \nu$ decays, they use slightly different ratios.
}

where $J_{\mu}^{q q^{\prime}}=\bar{q} \gamma_{\mu} q^{\prime}$ or $\bar{q} \gamma_{\mu} \gamma_{5} q^{\prime}$.

For instance, $R_{V_{0}}^{B \rightarrow D}$ yields $\left|h_{+}(1)\right|^{2}$ up to a multiplicative renormalisation, where $h_{+}(w)$ is the usual $B \rightarrow D \ell \nu$, heavy-quark form factor. The dependence of the square root of this ratio on $1 / m_{c}$ is shown in Figure' ${ }^{\prime} \bar{i}_{i}^{\prime}$ Statistical errors are indeed very small, and the deviation from 1 is significant statistically, which is certainly very encouraging. However, the tuning of the action and operators and the analysis of systematics in the hybrid approach is complex, and the results of $\left[4 \overline{4}_{4}^{\prime}, \overline{4} \overline{5}_{1}^{\prime}\right]$ should be confirmed by other groups.

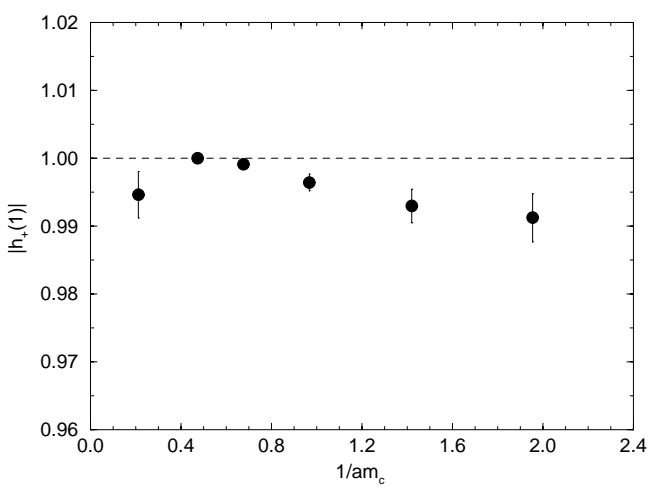

Figure 6: $R_{V_{0}}^{B \rightarrow D}$ of Eq. $(12.13)$ as a function of $1 / a m_{c}$ for an initial heavy quark with mass $a m_{b}=$ 2.11 [4]. The light quark's mass is that of the strange.

\subsection{2 $B \rightarrow D^{(*)} \ell \nu$ : recoil dependence}

While the shapes of $B \rightarrow D^{(*)} \ell \nu$ form factors obtained from the lattice are not required for determining $\left|V_{c b}\right|$ from these decays ${ }^{9}$, it is informative to compare these shapes to those determined by experiment. Such a comparison enables one to

\footnotetext{
${ }^{9}$ Extrapolation of the experimental data for the differential decay rate to the zero-recoil point with the help of a dispersive parameterisation such as the ones presented in Section 3.1 is adequate.
} 
verify the reliability of the extrapolation of experimental data to the zero-recoil point as well as to check the lattice method.

UKQCD [4흘 has presented preliminary results of a non-perturbatively $\mathcal{O}(a)$-improved calculation of a candidate Isgur-Wise function relevant for $B \rightarrow D^{(*)} \ell \nu$ decays:

$$
" \xi(w) " \equiv \frac{\left(1+\beta_{+}(1)\right)}{\left(1+\beta_{+}(w)\right)} \frac{h_{+}^{\text {lat }}(w)}{h_{+}^{\text {lat }}(1)},
$$

where $\beta_{+}(w)$ are the radiative corrections which match the QCD vector current, $\bar{c} \gamma^{\mu} b$, to its HQET counterpart and where $h_{+}^{\text {lat }}$ is the heavy-quark form factor $h_{+}$up to a multiplicative renormalisation. The ratio of Eq. (i2.1 $\overline{1}$ i) enforces nonperturbative lattice to continuum matching and should lead to cancellations of discretisation errors as well as subtract some zero-recoil power corrections to the heavy quark limit. Following $\left[4 \bar{T}_{1}, 4 \bar{u}_{i}\right]$, they study the heavy-quark mass dependence " $\xi(w)$ " for heavy-quark masses around the charm and find that it is consistent with 0 , in the range $1 \leq w \leq 1.2$. Thus, " $\xi(w)$ " is to a good approximation the Isgur-Wise function $\xi(w)$, at least in the above range of $w$, confirming the earlier results of $\left[4 \bar{\tau}_{1}, \bar{u}_{1}^{\prime} \overline{8}_{1}^{\prime}\right.$. The calculation is performed at two values of the lattice spacing, $\beta=6.2$ (finer) and 6.0 (coarser). Results for the Isgur-Wise function relevant for semileptonic $B \rightarrow D^{(*)}$ decays are shown in Figure $\overline{\bar{p}}_{1}$. Dependence on lattice spacing is small suggesting that discretisation errors are small. These results are compatible, yet more accurate than previous determinations (see $\left[\begin{array}{l}\overline{0} \\ \mathbf{B}\end{array}\right]$ ).

GOK are pursuing an NRQCD study of heavyto-heavy decays $[\overline{4} \overline{9}$. In addition to $B \rightarrow D$, they are also considering decays of the $B$ to radially excited $D$ mesons.

\subsubsection{Other decays}

UKQCD has investigated $\Lambda_{b} \rightarrow \Lambda_{c} \ell \nu$ and $\Xi_{b} \rightarrow$ $\Xi_{c} \ell \nu$ decays and determined the relevant IsgurWise functions [50

\section{Dispersive bounds}

The second part of my talk is concerned with the constraints obtained on weak matrix elements from the polarisation function

$$
\Pi_{J}^{\mu \nu}\left(q^{2}\right)=i \int d^{4} x e^{i q \cdot x}\left\langle 0\left|T\left\{J^{\mu}(x) J^{\nu \dagger}(0)\right\}\right| 0\right\rangle,
$$

where $J^{\mu}$ is chosen to be the operator which mediates the weak transition under consideration ${ }^{10}$.

I will further concentrate on two applications:

- model-independent constraints on $B \rightarrow D^{(*)} \ell \nu$ form factors used to eliminate the uncertainty in the extrapolation of experimental data to $w=1$ in the extraction of $\left|V_{c b}\right|$.

- model-independent extrapolations of heavyto-light lattice results obtained in a limited $q^{2}$ range.

$3.1 B \rightarrow D^{(*)} \ell \nu$

I will briefly summarise the methodology of dispersive constraints in the context of $B \rightarrow D \ell \nu$, with $J^{\mu}=V^{\mu}=\bar{c} \gamma^{\mu} b$. One first decomposes

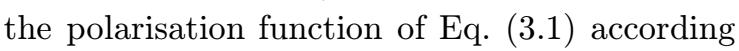
to helicity as

$\Pi_{V}^{\mu \nu}\left(q^{2}\right)=\left(q^{\mu} q^{\nu}-g^{\mu \nu} q^{2}\right) \Pi_{1^{-}}\left(q^{2}\right)+q^{\mu} q^{\nu} \Pi_{0^{+}}\left(q^{2}\right)$,

paralleling the decomposition of the matrix element $\left\langle D\left|V^{\mu}\right| B\right\rangle$ in terms of the form factors $f_{+}$ and $f_{0}$. To constrain $f_{0}$, one writes down a (once subtracted) dispersion relation for $\Pi_{0^{+}}$:

$$
\begin{aligned}
& \chi_{0^{+}}\left(q^{2}\right)=\frac{\partial}{\partial q^{2}}\left(q^{2} \Pi_{0^{+}}\left(q^{2}\right)\right) \\
& =\frac{1}{\pi} \int_{0}^{\infty} d t \frac{t \operatorname{Im}_{0^{+}}(t+i \epsilon)}{\left(t-q^{2}\right)^{2}},
\end{aligned}
$$

where the imaginary part of $\Pi_{0^{+}}$is essentially obtained by inserting a complete set of hadronic states between the vector currents in Eq. (3.1) :

$$
\begin{gathered}
\left.\cdots q^{\mu} q^{\nu}\right) \operatorname{Im}_{0^{+}}\left(q^{2}+i \epsilon\right)= \\
\frac{1}{2} \sum_{\Gamma}(2 \pi)^{4} \delta^{(4)}\left(q-p_{\Gamma}\right)\left\langle 0\left|V^{\mu}\right| \Gamma\right\rangle\left\langle\Gamma\left|V^{\nu \dagger}\right| 0\right\rangle .
\end{gathered}
$$

The scalar states which couple to $V^{\mu}$ are $|\Gamma\rangle=$ $\left|B_{c}\left(0^{+}\right)\right\rangle,\left|B_{c} \pi\right\rangle, \cdots,|B D\rangle, \cdots$, of which $|B D\rangle$ is the state of interest. The obvious positivity of the spectral function of Eq. (3. 3.4$)$ enables one to

\footnotetext{
${ }^{10}$ While the subject of inclusive heavy-quark sumrules is a fascinating one, it would take us too far afar.
} 

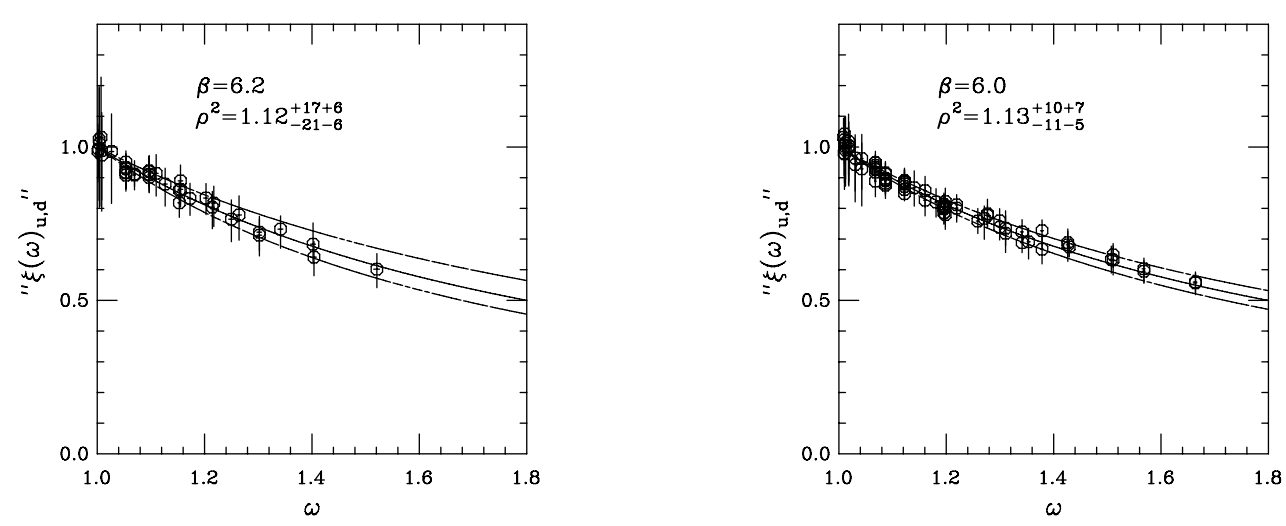

Figure 7: Candidate Isgur-Wise function relevant for $B \rightarrow D^{(*)} \ell \nu$ decays at two values of the lattice spacing (finer to the left) [46]. The curves, with statistical error bands, and the values of the zero-recoil slope, $\rho^{2}$, are the result of fits to $\xi(w)=\frac{2}{w+1} \exp \left[\left(2 \rho^{2}-1\right) \frac{1-w}{1+w}\right]$. The second error bar on $\rho^{2}$ is an estimate of discretisation errors as in [4]-1

write an upper bound on the weighted integral of the form factor $f_{0}$ along the cut induced by $|B D\rangle$ in $\Pi_{0^{+}}$:

$$
\chi_{0^{+}}\left(q^{2}\right) \geq \int_{-\infty}^{-1} d w k\left(w, q^{2}\right)\left|f_{0}(w)\right|^{2},
$$

where $k\left(w, q^{2}\right)$ is a kinematical function and where $w=\left(M_{B}^{2}+M_{D}^{2}-t\right) /\left(2 M_{B} M_{D}\right)$. This bound is interesting because the function $\chi_{0^{+}}\left(q^{2}\right)$ can be computed in QCD in terms of an expansion in $\alpha_{s}$ and condensates, for $q^{2} \ll m_{b}^{2}$ (i.e. well below the lowest $\left|B_{c}\left(0^{+}\right)\right\rangle$state). Of course, the more states $|\Gamma\rangle$ one can include in Eq. (13.4) in a model-independent way, the better the bounds on the form factor $f_{0}$. In particular, one can use heavy-quark-spin symmetry to include the contributions of $\left|B^{*} D\right\rangle,\left|B D^{*}\right\rangle^{11}$ and $\left|B^{*} D^{*}\right\rangle$, in the semileptonic domain.

Translating the bound of Eq. (3.5i) into a bound on $f_{0}$ in the semileptonic region is an exercise in complex analysis. For convenience, one performs the conformal transformation

$$
z(w, a)=\frac{\sqrt{w+1}-\sqrt{2} a}{\sqrt{w+1}+\sqrt{2} a} ; \quad a>0,
$$

which maps the cut plane in $w$ onto the unit $\operatorname{disc}^{12}$. Then the bound of Eq. (3.5) becomes

$$
\chi_{0^{+}}\left(q^{2}\right) \geq \oint \frac{d z}{2 \pi i z}\left|\phi\left(z, q^{2}\right) f_{0}(z)\right|^{2},
$$

\footnotetext{
${ }^{11}\left|B D^{*}\right\rangle$ does not actually contribute to the $0^{+}$channel.

${ }^{12}$ For $a=1$, the zero-recoil point $w=1$ gets mapped onto the origin $z=0$.
}

where $\phi\left(z, q^{2}\right)$ is the conformally mapped version of $k\left(w, q^{2}\right)$, with kinematical singularities inside the unit disc removed. This bound implies:

- elliptic constraints on the slope, curvature and higher derivatives of $f_{0}(z)$ at $z=0$; we show such constraints on $f_{+}(z)$, related to $f_{0}(z)$ by heavy-quark-spin symmetry near $z=0$, in Figure $\underline{\underline{p}} \cdot$

- that the remainder of the expansion of $\phi f_{0}(z)$ in $z$, once subthreshold singularities are accounted for, can be bounded and that the expansion converges rapidly, as was first remarked in [51].

The two most complete analyses are those of [53 are also analysed. One of the upshot of these analyses is that the form factors for $B^{(*)} \rightarrow D^{(*)}$ vector and axial transitions can each be described with an accuracy better than $2 \%$ with one parameter and an overall normalisation.

For instance, [52] gives for the form factor which determines $B \rightarrow D \ell \nu$ decays,

$$
\begin{gathered}
\frac{\mathcal{F}_{D}(w)}{\mathcal{F}_{D}(1)}=\frac{f_{+}(w)}{f_{+}(1)} \approx 1-8 \rho_{1}^{2} z+\left(51 . \rho^{2}-10 .\right) z^{2} \\
-\left(252 . \rho^{2}-84 .\right) z^{3}
\end{gathered}
$$

with $-0.17<\rho^{2}<1.51$ and $z=(\sqrt{w+1}-$ $\sqrt{2}) /(\sqrt{w+1}+\sqrt{2})$. The bounds on $\rho^{2}$ are slighlty stronger than those given in Figure in [52]. Similar parameterisations were given for $\mathcal{F}_{D^{*}}(w)$. 


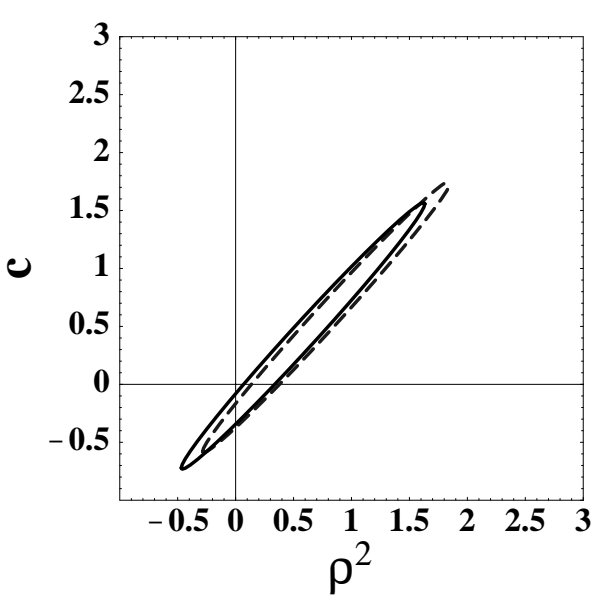

Figure 8: Bounds on the parameters $\rho^{2}$ and $c$ which appear in $f_{+}(w)=f_{+}(1)\left(1-\rho^{2}(w-1)+c(w-1)^{2}+\right.$ ...) [52]. Only points within the ellipses are allowed. The bounds were obtained in the $0^{+}$channel. They take into account contributions, amongst others, from the $|B D\rangle,\left|B^{*} D\right\rangle$ and $\left|B^{*} D^{*}\right\rangle$ states, including (solid) and not including (dashed) corrections to heavy-quark spin symmetry.

These dispersive parameterisations have been tried by experimental collaborations [54] and describe the data very well. They eliminate the uncertainty in the extrapolation of the differential decay from $w \neq 1$ to the zero-recoil point.

\section{$3.2 B \rightarrow \pi \ell \nu$ and related work}

The first application of dispersive bounds to $B \rightarrow$ $\pi \ell \nu$ was performed in [5 55$]$ where it was argued that these techniques could be used to eliminate certain models for the relevant form factors. In [14], these techniques were extended to extrapolate, to the full kinematical range, lattice results for $B \rightarrow \pi \ell \nu$ form factors obtained at high $q^{2}$. The resulting lattice-constrained bounds are shown in Figure agreement is excellent.

Similar bounds were obtained for the total rate and are summarized in Table $\overline{4}_{r}^{1}$ Since the systematic uncertainties added to the lattice re-

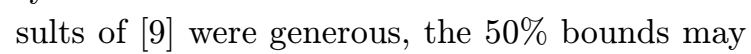
be a reasonable representation of the constraints given by the dispersive method.

The results of [14i] are rather old and can presumably be improved with the lattice results and additional constraints. For instance, the au-

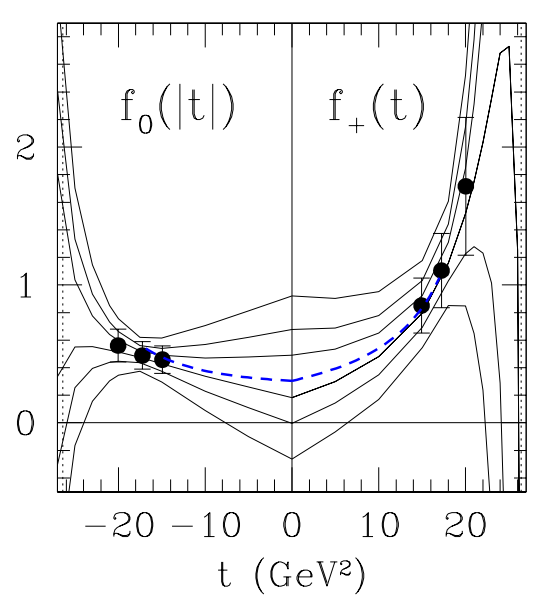

Figure 9: Dispersive bounds for $f_{0}(|t|)$ and $f_{+}(t)$ in $B^{0} \rightarrow \pi^{-} \ell^{+} \nu$ decays [1. The points are the lattice results of $[9]$ with added systematic errors. The pairs of fine curves are, from the outermost to the innermost, the $95 \%, 70 \%$ and $30 \%$ bounds, where percentages represent the likelihood that the form factor take a value between the corresponding pair of curves at the given $t$. The dashed curves are the LCSR results of [1] 1 in].

\begin{tabular}{ccc}
\hline$\Gamma\left(B^{0} \rightarrow \pi^{-} \ell^{+} \nu\right)$ & $f_{+}(0)$ & $\mathrm{CL}$ \\
\hline $4.8 \rightarrow 10$ & $0.18 \rightarrow 0.49$ & $30 \%$ \\
$4.4 \rightarrow 13$ & $0.10 \rightarrow 0.57$ & $50 \%$ \\
$3.6 \rightarrow 17$ & $0.00 \rightarrow 0.68$ & $70 \%$ \\
$2.4 \rightarrow 28$ & $-0.26 \rightarrow 0.92$ & $95 \%$ \\
\hline
\end{tabular}

Table 4: Bounds on the rate in units of $\left|V_{u b}\right|^{2} p s^{-1}$ and on $f_{+}(0)$ [ist].

thors of $\left[\overline{5}^{5} \overline{6}_{1}\right]$ discuss the inclusion of soft-pion constraints. The authors of [57] discuss the improved constraints brought about by considering higher moments of the dispersion relations discussed above, as well as the inclusion of the $B^{*} \rightarrow \pi \ell \nu$ through the use of heavy-quark spin symmetry.

The application of these techniques to $B \rightarrow$ $\rho \ell \nu$ and $B \rightarrow K^{*} \gamma$ decays is investigated in [5ㅎ․] and [59.

\section{Conclusion}

\subsection{Lattice}

Many new lattice results for semileptonic decays of the $B$ have or are about to appear. They 
have significantly higher statistics than first generation calculations. This additional statistical accuracy has enabled some groups to perform the difficult extrapolation to the physical pion mass in $B \rightarrow \pi \ell \nu$ decays, and reconstruct the $q^{2}$-behaviour of the physical form factors without $S U(3)$-flavour assumptions, for $q^{2} \gtrsim 15 \mathrm{GeV}^{2}$. Moreover, some of these calculations are nonperturbatively $\mathcal{O}(a)$-improved indicating that they have reduced discretisation and matching errors. Others have been performed for many lattice spacings, which will enable extrapolations to the physical continuum or, at least, a better quantification of discretisation errors. Most of these results, unfortunately, are still obtained in the unphysical quenched approximation. However, a number of groups are beginning to partially include the effect of fermion loops on these decays and preliminary results were presented at Lattice 99. Furthermore, more and more theoretical constraints on the $q^{2}$-behaviour of the relevant form factors, such as those described in Section ' 2.2 .1 , are being taken into account. This should enable, an extension of the kinematical range covered by lattice calculations.

Many new results are also appearing for semi-

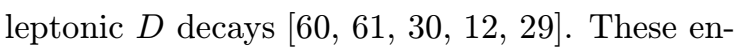
able a calibration of the lattice method through comparison with experiment. As suggested in [600], the ratio of partial widths for $D \rightarrow \pi \ell \nu$ and $D \rightarrow K \ell \nu$ is also a good way to reduce the uncertainty on $\left|V_{c d}\right| /\left|V_{c s}\right|$, currently $\sim 17 \%$. Furthermore, the ratio of differential decay rates for $B \rightarrow \rho(\pi) \ell \nu$ to those for the corresponding $D$ decays may provide a means of determining $\left|V_{u b}\right|$ with reduced theoretical uncertainties [1] $\left.{ }^{2}\right]$.

\subsection{Dispersive bounds}

Dispersive bounds for heavy-to-light decays have not been worked on in a while and can presumably be improved with new lattice results and additional constraints.

For $B \rightarrow D^{(*)} \ell \nu$ decays, dispersive bounds are quite mature and improvement on current results seems difficult.

\section{Acknowledgments}

Warm thanks to L. Del Debbio, J. Flynn, V.
Lesk, C. Maynard, J. Nieves and other colleagues of UKQCD for fruitful collaborations and discussions. I also thank D. Becirevic, C. DeTar, G. Douglas, S. Hashimoto, J. Hein, A. Kronfeld, T. Onogi and S. Ryan for their help in putting together this review.

\section{References}

[1] Shoji Hashimoto, these proceedings.

[2] S. Hashimoto, hep-lat/9909136.

[3] J.M. Flynn and C.T. Sachrajda, heplat/9710057.

[4] J.M. Flynn, hep-lat/9710080.

[5] C. Bernard et al., hep-lat/9909076.

[6] B.H. Behrens et al. [CLEO Collaboration], hepex/9905056; L. Gibbons, private communication.

[7] A. Abada et al., Nucl. Phys. B416 (1994) 675.

[8] C.R. Allton et al. [APE Collaboration], Phys. Lett. B345 (1995) 513.

[9] D.R. Burford, H.D. Duong, J.M. Flynn, J. Nieves, B.J. Gough, N.M. Hazel and H.P. Shanahan [UKQCD Collaboration], Nucl. Phys. B447 (1995) 425.

[10] K.C. Bowler et al. [UKQCD Collaboration], hep-lat/9911011.

[11] S. Ryan, A. El-Khadra, S. Hashimoto, A. Kronfeld, P. Mackenzie and J. Simone, Nucl. Phys. Proc. Suppl. 73, 390 (1999) hep-lat/9810041.

[12] S.M. Ryan, A.X. El-Khadra, A.S. Kronfeld, P.B. Mackenzie and J.N. Simone, heplat/9910010.

[13] S. Hashimoto, K. Ishikawa, H. Matsufuru, T. Onogi and N. Yamada, Phys. Rev. D58 (1998) 014502.

[14] L. Lellouch, Nucl. Phys. B479 (1996) 353.

[15] P. Ball, JHEP 09 (1998) 005.

[16] V.M. Belyaev, A. Khodjamirian and R. Ruckl, Z. Phys. C60 (1993) 349.

[17] V.M. Belyaev, V.M. Braun, A. Khodjamirian and R. Ruckl, Phys. Rev. D51 (1995) 6177.

[18] A. Khodjamirian, R. Ruckl, S. Weinzierl and O. Yakovlev, Phys. Lett. B410 (1997) 275.

[19] E. Bagan, P. Ball and V.M. Braun, Phys. Lett. B417 (1998) 154. 
[20] A. Khodjamirian, R. Ruckl and C.W. Winhart, Phys. Rev. D58 (1998) 054013.

[21] L. Del Debbio, J.M. Flynn, L. Lellouch and J. Nieves [UKQCD Collaboration], Phys. Lett. B416 (1998) 392.

[22] D. Becirevic and A.B. Kaidalov, hep$\mathrm{ph} / 9904490$.

[23] D. Becirevic, P. Boucaud, J.P. Leroy, V. Lubicz, G. Martinelli, F. Mescia and F. Rapuano, Phys. Rev. D60 (1999) 074501.

[24] L. Wolfenstein, Phys. Lett. B291 (1992) 177.

[25] G. Burdman and J.F. Donoghue, Phys. Lett. B280 (1992) 287.

[26] J. Charles, A. Le Yaouanc, L. Oliver, O. Pene and J.C. Raynal, Phys. Rev. D60 (1999) 014001.

[27] V.L. Chernyak and I.R. Zhitnitsky, Nucl. Phys. B345 (1990) 137.

[28] A. Ali, V.M. Braun and H. Simma, Z. Phys. C63 (1994) 437 hep-ph/9401277.

[29] A. Abada, D. Becirevic, P. Boucaud, J.P. Leroy, V. Lubicz, G. Martinelli and F. Mescia, heplat/9910021.

[30] K.C. Bowler et al. [UKQCD Collaboration], hep-lat/9910020.

[31] G.M. de Divitiis, L. Del Debbio, M. Di Pierro, J.M. Flynn, C. Michael and J. Peisa [UKQCD Collaboration], JHEP 10 (1998) 010.

[32] R. Casalbuoni, A. Deandrea, N. Di Bartolomeo, R. Gatto, F. Feruglio and G. Nardulli, Phys. Rept. 281 (1997) 145.

[33] I.W. Stewart, Nucl. Phys. B529 (1998) 62.

[34] J.M. Flynn et al. [UKQCD Collaboration], Nucl. Phys. B461 (1996) 327.

[35] N. Isgur and M.B. Wise, Phys. Lett. B237 (1990) 527.

[36] G. Burdman and J.F. Donoghue, Phys. Lett. B270 (1991) 55.

[37] P.A. Griffin, M. Masip and M. McGuigan, Phys. Rev. D50 (1994) 5751.

[38] P. Ball and V.M. Braun, Phys. Rev. D58 (1998) 094016

[39] M. Ciuchini, E. Franco, G. Martinelli, L. Reina and L. Silvestrini, Phys. Lett. B334 (1994) 137.

[40] CLEO Collaboration, R. Ammar et al., CLEO CONF 96-05; CLEO Collaboration, S. Glenn et al., CLEO CONF 98-17.
[41] R. Gupta and T. Bhattacharya, Nucl. Phys. Proc. Suppl. 47 (1996) 473.

[42] A. Abada et al. [APE Collaboration], Phys. Lett. B365 (1996) 275.

[43] C. Bernard, P. Hsieh and A. Soni, Phys. Rev. Lett. 72 (1994) 1402.

[44] S. Hashimoto, A.X. El-Khadra, A.S. Kronfeld, P.B. Mackenzie, S.M. Ryan and J.N. Simone, hep-ph/9906376.

[45] J.N. Simone, S. Hashimoto, A.X. El-Khadra, A.S. Kronfeld, P.B. Mackenzie and S.M. Ryan, hep-lat/9910026.

[46] G. Douglas [UKQCD Collaboration], heplat/9909126 and Ph. D. Thesis, The University of Edinburgh, 1999.

[47] S.P. Booth et al. [UKQCD Collaboration], Phys. Rev. Lett. 72 (1994) 462.

[48] K.C. Bowler et al. [UKQCD Collaboration], Phys. Rev. D52 (1995) 5067.

[49] J. Hein, P. Boyle, C.T. Davies, J. Shigemitsu and J. Sloan [UKQCD Collaboration], heplat/9908058.

[50] K.C. Bowler et al. [UKQCD Collaboration], Phys. Rev. D57 (1998) 6948.

[51] C.G. Boyd, B. Grinstein and R.F. Lebed, Phys. Lett. B353 (1995) 306.

[52] I. Caprini, L. Lellouch and M. Neubert, Nucl. Phys. B530 (1998) 153.

[53] C.G. Boyd, B. Grinstein and R.F. Lebed, Phys. Rev. D56 (1997) 6895.

[54] See, for instance, J. Bartelt et al. [CLEO Collaboration], Phys. Rev. Lett. 82 (1999) 3746.

[55] C.G. Boyd, B. Grinstein and R.F. Lebed, Phys. Rev. Lett. 74 (1995) 4603.

[56] T. Mannel and B. Postler, Nucl. Phys. B535 (1998) 372.

[57] C.G. Boyd and M.J. Savage, Phys. Rev. D56 (1997) 303.

[58] D. Becirevic, Phys. Rev. D54 (1996) 6842.

[59] D. Becirevic, hep-ph/9707271.

[60] J.N. Simone, A.X. El-Khadra, S. Hashimoto, A.S. Kronfeld, P.B. Mackenzie and S.M. Ryan, Nucl. Phys. Proc. Suppl. 73 (1999) 393.

[61] C.M. Maynard [UKCQD Collaboration], Nucl. Phys. Proc. Suppl. 73 (1999) 396. 